\title{
Intelligent Space environment for ethorobotics
}

\author{
Balázs Nagy \\ MOGI Department \\ Budapest University of Technology and Economics \\ Budapest, Hungary \\ nagybalazs@mogi.bme.hu
}

\author{
Štefan Gubo \\ Department of Mathematics and Informatics \\ J. Selye University \\ Komárno, Slovakia \\ gubos@ujs.sk
}

\begin{abstract}
This paper presents a setup using a mobile robot agent in an intelligent space. The proposed concept based on a holonomic driving system mount on the robot. Due to the driving system different moving behaviours and path planning algorithms can be tested. To examine the robot movements and provide the needed support the robot agent was placed in a Motion capture system. The special environment around the robot is a motion track system which can track the movements of agents in its space via markers.
\end{abstract}

Keywords-intelligent space; etorobotics; motion capture system

\section{INTRODUCTION}

Nowadays more and more robots are constructed due to the high level of automatization. Robots are part of the everyday life, which means that interactions between robots and humans has been increased significantly in the recent years. This trend will continue in the future, so a new field of study, called ethorobotics, has emerged [1]. The main aim of this field of study is to define behaviour models, which can be implemented on mobile robots. With the help of these models the behaviour of the robots can be more naturalistic. The distant goal is to integrate the robots into the society which means even non-programmer humans can communicate with robots without any specific acknowledgement. Narrowing the communication gap between humans and robots and increasing the number of interactions between the two brings us a step closer to our cooperative future.

This paper structure is the following: In Section II a robot agent called Ethon is being introduced. This section summarizes the main specification of the robot. In Section III the concept of intelligent space is being introduced briefly. Section IV summarizes the information about the used motion capture system. Section V contains the final conclusion and the possible new development directions.

\section{ETHON}

\section{A. Driving system}

Nature inspired robots based on a similarity with animals or humanoids. These constructions use legs, as a primary moving system. Mayor disadvantages of humanoid robots are the high cost of the leg mechanism and the complexity of the control system. Balancing a robot is a challenging problem and computationally expensive, this requires a powerful processing unit. In the other hand wheel based construction can be more stable. The control system is less complex. Using wheels means the degree of freedom has reduced. In order to maintain as many degrees of freedom as possible, we suggest using omnidirectional driving system with holonomic wheels. The holonomic wheel of the robot can be seen in Figure 1. Proper using of holonomic wheels demand a detailed model [2].

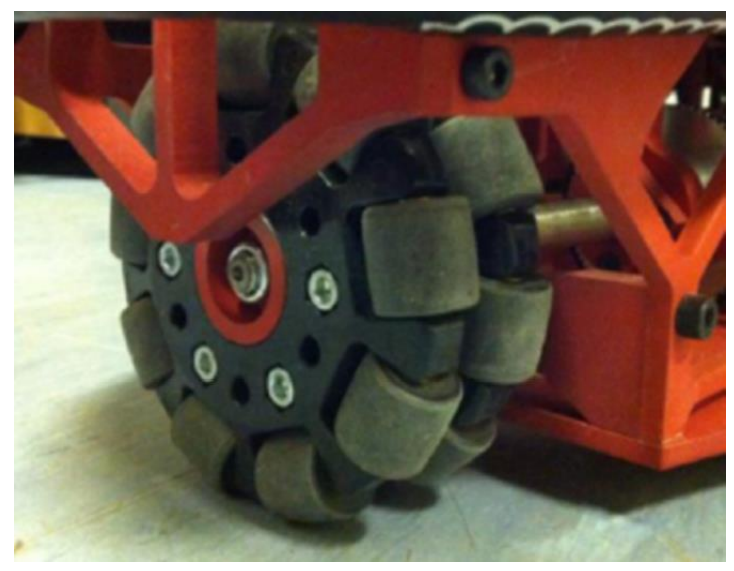

Figure 1: Holonomic wheel

Moving is a key element in behaviour models. Differential driving robots can go only in one direction, where the robot faces. While avoiding an obstacle these robots have to turn away from the obstacle and face the direction of the evasive manoeuvre. Animal like creatures can avoid an obstacle while constantly keep the obstacle in sight or facing the desired end position regardless of the direction of the evasive path.

\section{B. Robot concept}

Ethon is the robot agent used during the ethorobotic studies. The concept of the robot is shown in Figure 2. Ethon has an omnidirectional drive. The holonomic wheels provide the ability to move in any direction without change its orientation. In this way the movements of a robot can be more expressive 


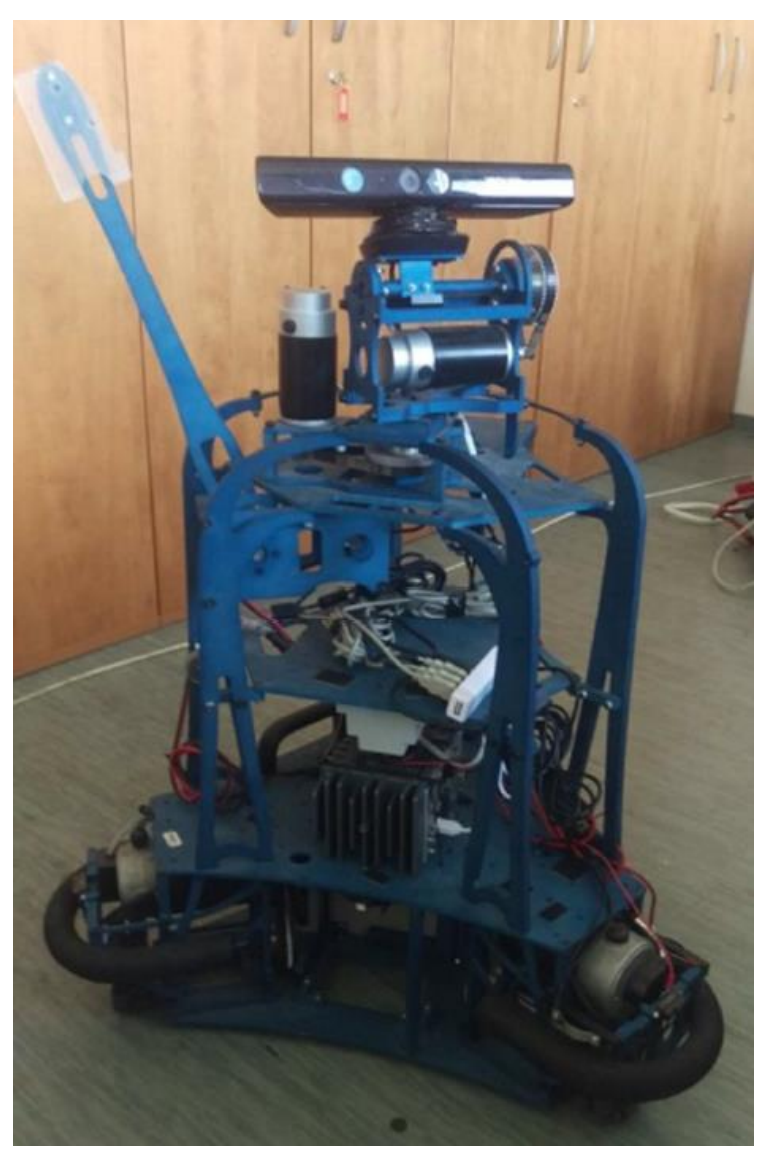

Figure 2: Ethon

\section{Description of Guiding Style Models}

One of the widely adopted guiding style models is the potential based guiding (PBG) [2]. The robot can detect objects in the scanned area (Figure 3 ). The scanned area is divided into $n$ scanned lines that are pointed into directions of $\vec{e}_{z}$ (unique vectors, where $z=1 \ldots n$ ). The radial scanned lines structure has an important advantage that they density is growing with the decreasing distance to the robot. The sensor system provides the distance between the robot and the object on the scanned lines. The main idea of the potential based guiding is to repulse (or attract) the robot from/to the obstacles. The objects and the target generate imaginary forces acting on the robot. Summing the effect of these virtual forces, the desired moving direction can be obtained. The virtual vectors must be calculated for each location as quickly as possible to achieve a smooth and reactive guiding.

The magnitudes of the repulsive forces are usually inversely proportional to the distance between the obstacles and the vehicle but they can be described by any non-linear functions.
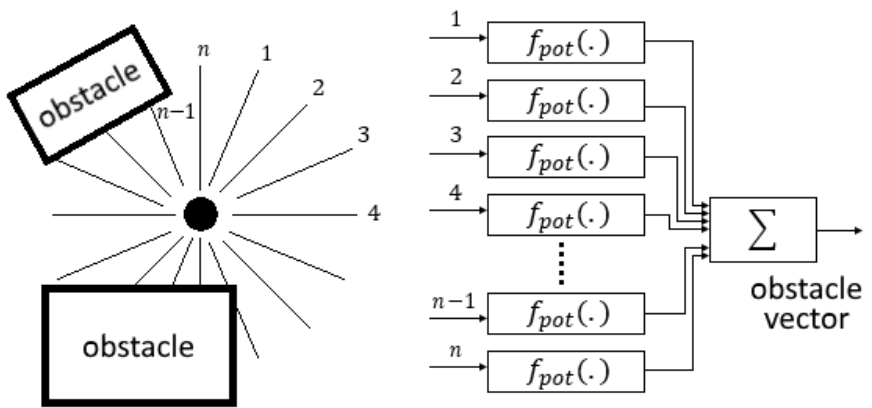

Figure 3: Potential field obstacle avoidance concept

In many applications the same formula is used to compute all the repulsive forces, resulting a symmetrical potential field. In order to achieve different guiding styles asymmetrical potential fields may be used as well.

Objects often mean protection or hiding for animals. These are not just obstacles which have to be bypassed. Most visibly, cats often go close to the wall even if it results in a longer path as demonstrated in Figure 4 [3]. This antipredator behaviour specific to small mammals is well described by ethological studies. It is affected by anxiety-related medicines, therefore medical companies also analyse it in the widely used animal tests called open field test [4].

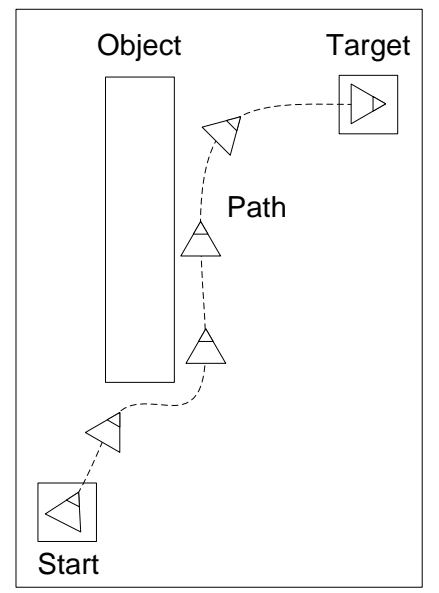

(a)

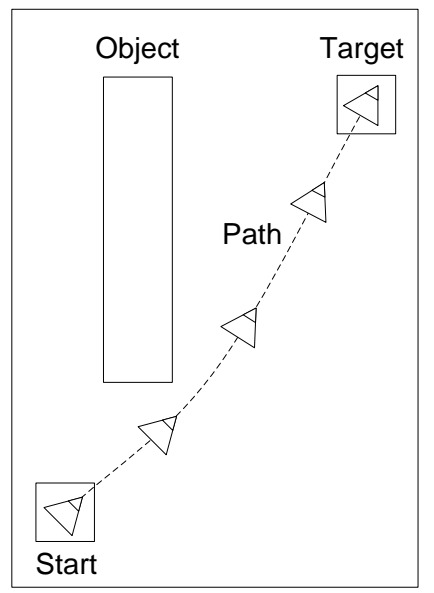

(b)
Figure 4: The path (a) with and (b) without the object proximity-seeking motion characteristics. This nature is specific especially for small mammals, like cats or rodents, as they often stay close to objects for hiding and protection.. [3]

\section{INTELLIGENT SPACE}

In the recent years several independent groups have conducted studies relating to Intelligent Space. Some of the connecting projects are the following.

\section{A. Ada-the intelligent space [5]}

Ada is an artificial, avantgarde organism, a creature in the shape of a space which can perceive and via sounds, light and projections expressing its inner states. Ada is based upon the 
newest achievements in neuroscience. In contrast to conventional computer systems which are rule based, Ada is a neural network resembling our brain: Ada can learn and is "unpredictable". Ada's actions correspond to emotional behavioural patterns. Like humans or animals Ada expresses its being through coordinated goal-oriented behaviour, which is controlled by its own will. The architecture and dramaturgy of Ada are designed in a way that visitors will experience Ada as a novel synthetic body. They will have the possibility to explore Ada step by step. The encounter with Ada is an experiment, comparable to every approach between two beings who try - driven by a natural impulse - to explore each other to gain security and sovereignty.

\section{B. Intelligent Space Oxygen Project [6]}

Space-centered computation embedded in ordinary environments defines intelligent spaces populated by cameras, microphones, displays, sound output systems, radar systems, wireless networks, and controls for physical entities such as curtains, lighting, door locks, soda dispensers, toll gates, and automobiles. People interact in intelligent spaces naturally, using speech, gesture, drawing, and movement, without necessarily being aware that computation is present. Humancentered computation supports social interaction in its familiar context. People communicate with computation easily, as they do with each other, using shared knowledge and intelligence. Freely-available computation makes it easy for people to monitor and control their environment. Physical objects become immensely more useful and usable, and people and agents in other environments become immensely easier to reach.

\section{Intelligent Space Robotics [7]}

Bold missions of exploration, discovery and the development of space demand cognitive robots and reliable autonomous spacecraft. Scenarios in which robots operate with minimal Earth communication are essential to this future and compel autonomy. The mission successes are envisioned through robots with competence that leaps beyond current state-of-art, and perception and cognition capabilities that are manifested and evolved over long, productive robot lifetimes. Robots will be the agents of planetary surface and deep space exploration, handling the repetitive and time-consuming tasks of data collection and data reduction. Teams of robots will survey vast regions, cataloguing and classifying geological features and formations and searching for evidence of life, all the while tailoring their investigations to what they discover and seeking opportunities to discover more. Robots will be humankind's agents and partners in space, constructing and servicing orbital and surface facilities with millions of components. They will operate on distant planets, using highlevel directives, responding to and interacting with humans. This approach to achieving this vision is research, education, and manifestation of intelligent space robotics through an Intelligent Space Robotics Center. The Centre will perform breakthrough research in robotics technology relevant to
NASA missions and will deliver the next generation of space roboticists.

\section{Intelligent Space for manufacturing [8]}

This project proposed a new paradigm for robot teaching and supervising which is based on cognitive infocommunication channels for human-machine interaction. The robot is considered as an unskilled worker "who" (which) is strong and capable for precise manufacturing. "He" (it) has a special kind of intelligence, but "he" is handicapped in some senses, that is why "he" needs special treatment. We must command "him" clearly in a special way and we must supervise "his" work. If we can elaborate a proper way for communicating with this "new worker", as an additional dimension of robotization, we can get a capable new "colleague". The ultimate goal is to help the boss to be able to give the daily task to a robot in a similar way as he/she able to give the jobs to the human workers. For example, by providing the CAD documentation with some additional verbal explanation.

\section{E. iSpace concept}

Concept of the iSpace based on the idea that computationally expensive algorithms can be outsourcing from the onboard computer of the robot into the environment of the robot [9]. In this setup there is no need a powerful computer with high computation capacity onboard. In this case the energy consumption can be maintained in a lower level on the mobile robot so the battery life increases. The iSpace is an intelligent environment. In order to observe the dynamic environment, many intelligent devices, which are called Distributed Intelligent Network Devices, are installed into the room as shown in Figure 5.

The DIND is a fundamental element of the iSpace. It consists of three basic components including sensors, processors and network devices. By communicating with each DIND [10], iSpace can perceive and understand events in the whole space.

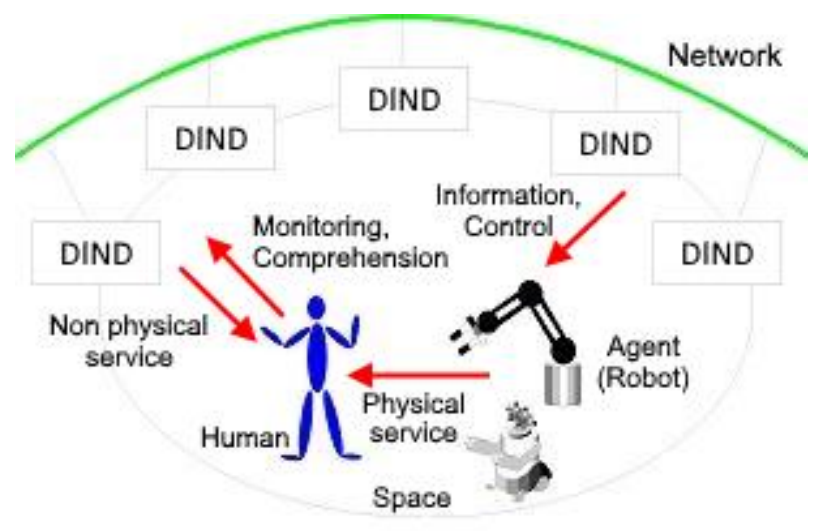

Figure 5: Concept of the Intelligent Space

\section{MOTION CAPTURE SYSTEM}

Running a behaviour engine and processing images at once is a very resource consuming progress. To relieve the main 
onboard computer of the robot we moved some of the algorithms off the robot and generated an intelligent space concept around it. To do so a motion capture system was built up [11]. The system architecture can be seen in Figure 6.

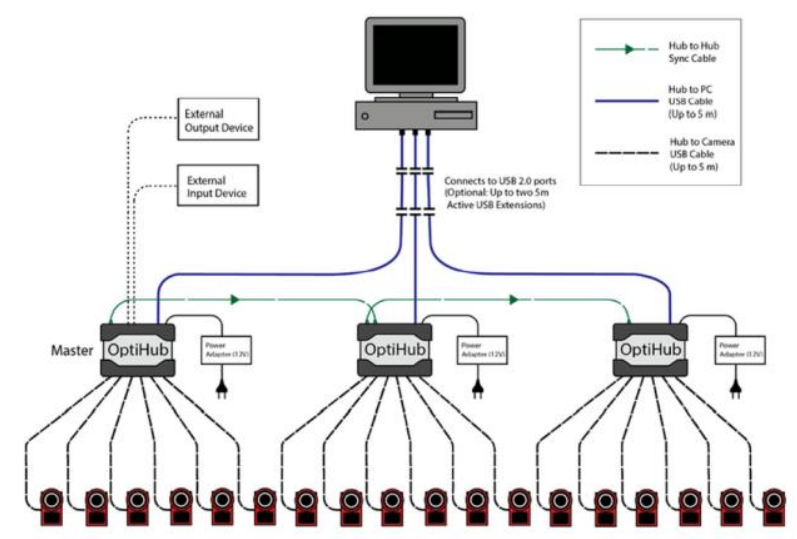

Figure 6: Motion capture system architecture

\section{A. Calculation of the optimal camera positions}

The system contains 18 cameras. Each camera has its own image processing unit and calculates the position of the seen markers. To determine the different markers position and track them in 3D space the system uses epipolar geometry. Avoid ambiguous correspondences at least 3 cameras are required. According to three pictures from three different cameras the position of the questionable marker can be calculated.

\section{B. The Problem}

While installing a motion caption (MOCAP) system the main problem is how to arrange cameras. With fix focus cameras data of the arrangement are the optical centers $\left(x_{i}, y_{i}, z_{i}\right)$ and Euler angles $\left(\alpha_{i}, \beta_{i}, \gamma_{i}\right)(i \in[1, N])$ of $N$ cameras. Let $V$ be the active volume of the examination. There is a requirement that at all $(\xi, \eta, \zeta)$ points of $V$ are seen by minimum $n$ cameras. Let $f$ be the observed space the function of camera positions. Every $(\xi, \eta, \zeta)$ point of the space can associated with a number $(g)$ showing how many cameras watch that very point.

The conditional optimum problem is

$$
\begin{gathered}
V=\max f\left(x_{1}, y_{1}, z_{1}, \alpha_{1}, \beta_{1}, \gamma_{1}, \cdots x_{N}, y_{N}, z_{N}, \alpha_{N}, \beta_{N}, \gamma_{N}\right) \\
g(\xi, \eta, \zeta)>n \quad(\xi, \eta, \zeta) \in V
\end{gathered}
$$

Evidently the explicit form and solution in closed form of (1) does not exist. The values of $f$ function fitting the condition can be generated, so the extremum can be searched for.

Usually cameras are attached to a rail on the wall. It is a usual to require that at least 3 cameras should watch points of the examined space. In this case $f$ function in equation (1) is simpler. In place of $\left(x_{i}, y_{i}, z_{i}\right)$ coordinates come $l_{i}$ coordinate, the distance of the $i$ th camera from a selected origin measured on the rail.

$$
\begin{gathered}
V=\max _{g(\xi, \eta, \zeta)>3} f\left(l_{1}, \alpha_{1}, \beta_{1}, \gamma_{1}, \cdots l_{N}, \alpha_{N}, \beta_{N}, \gamma_{N}\right) \\
(\xi, \eta, \zeta) \in V
\end{gathered}
$$

The simplest case of this issue is, when the subjects of the examination are ignored, only the measured space is maximized. In case the measured objects are also considered then the $g$ function is modified. Positions of subjects are discretized, and the hiding is considered.

The concrete test was executed in a room sized $10 \times 7 \times 4.4 \mathrm{~m}$ by 18 cameras on a rail positioned in $3 \mathrm{~m}$ height. The maximum examined volume was $6 \times 5 \times 3 \mathrm{~m}$ (Figure 7).

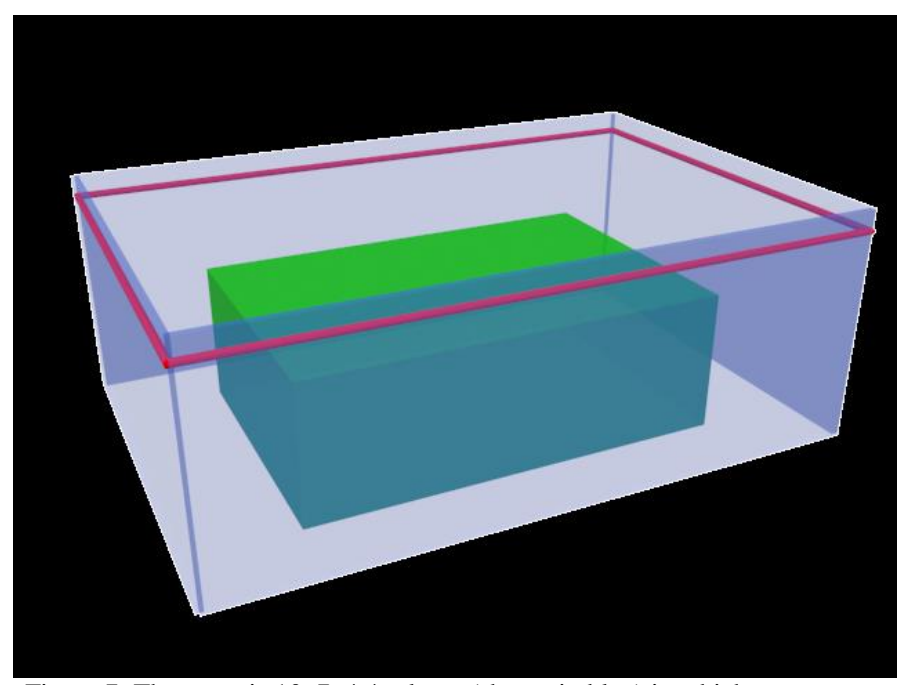

Figure 7: The room is $10 \times 7 \times 4.4 \mathrm{~m}$ large (shown in blue) in which we want to track the markers in the center $6 \times 5 \times 3 \mathrm{~m}$ volume (shown in green). The cameras are attached to a rail system on the wall of the room (shown in red).

Figure 8 shows the mounted motion capture system and the mobile agent.

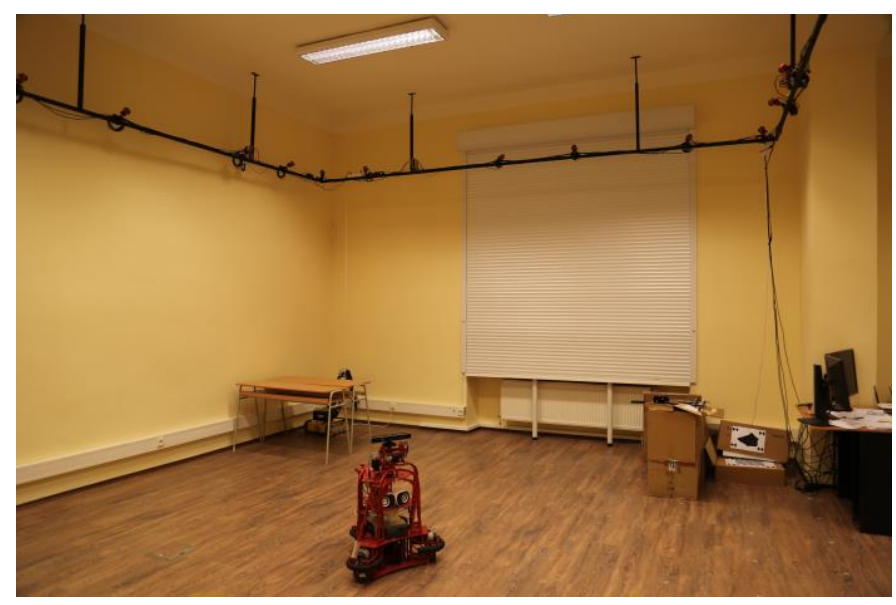

Figure 8: Mobil agent in iSpace

\section{Results}

As a result of this study we managed to build up a motion capture laboratory which can track multiple agents in real 
time. The motion tracking softer broadcasting the collected information to a multi cast group via wireless connection. In this way the agents presented in the intelligent space has access to all of the information. An agent can send a request command to the motion tracking server to get the position and orientation date of their own or any other agent in the space.

With the help of this laboratory we can examine human robot or even robot-animal interactions. Tests, focusing on the connection between the motion tracking server and the robot agents, are in progress and the results will be discussed in our next paper.

\section{CONCLUSION-FUTURE PLANS}

As a conclusion we can say that our group of researchers managed to set up a motion capture system which can be used as an intelligent space in the future. The mobile agent, called Ethon, is also functional. Mounting up this research environment we opened a path before various ethorobotical and ethological experiment. In the future specific experiments can be take place in this iSpace concept.

\section{ACKNOWLEDGMENT}

This work was partially supported by the Research \& Development Operational Programme for the project "Modernization and Improvement of Technical Infrastructure for Research and Development of J. Selye University in the Fields of Nanotechnology and Intelligent Space", ITMS 26210120042, co-funded by the European Regional Development Fund

\section{REFERENCES}

[1] A. Miklosi, P. Korondi, V. Matellan and M. Gacsi, "Ethorobotics: A New Approach to Human-Robot Relationship," FRONTIERS IN PSYCHOLOGY, vol. 8, no. 958, pp. 1-8, 2017.

[2] S. Mizik, P. Baranyi, P. Korondi and M. Sugiyama, "Virtual Training of Vector Function based Guiding Styles," Transactions on Automatic Control and Computer Science, vol. 46(60), no. 1, pp. 81-86, 2001.

[3] B. Kovács, G. Szayer, F. Tajti, M. Burdelis and P. Korondi, "A novel potential field method for path planning of mobile robots by adapting animal motion attributes," Robotics and Autonomous Systems, vol. 82, pp. 24-34, 2016.

[4] D. Lipkind, A. Sakov, N. Kafkafi, G. Elmer, Y. Benjamini and I. Golani, "New replicable anxiety-related measures of wall vs center behavior of mice in the open field," J. Appl. Physiol, vol. 97, p. 347-359, 2004.

[5] K. Eng, A. Babler, U. Bernardet, M. Blanchard, A. Briska, J. Conradt, M. Costa, T. Delbruck, R. Douglas, K. Hepp, D. Klein, J. Manzolli, M. Mintz, T. Netter, F. Roth, U. Rutishauser, K. Wassermann, A. Whatley, A. Wittmann, R. Wyss and P. Verschure, "Ada: constructing a synthetic organism," in IEEE/RSJ International Conference on Intelligent Robots and Systems, Lausanne, Switzerland, 2002.

[6] M. Dertouzos, "The Oxygen Project," Scientific American, vol. 282, no. 3, p. 52-63, 1999.

[7] E. Sato and T. Yamaguchi, "HUMAN TO ROBOTS COMMUNICATION USING ONTOLOGICAL NETWORK SYSTEM," International Journal of Innovative Computing, Information and Control, vol. 2, no. 1, 2006.

[8] C. M. Horváth and S. Kovács, "New cognitive infocommunication channels for human-machine interaction," Recent Innovations in Mechatronics (RIiM), vol. 4, no. 1, pp. 1-8, 2017.

[9] P. Korondi and H. Hashimoto, "Intelligent space, as an integrated intelligent system," in Proceedings of the 15th International Conference on Electrical Drives and Power Electronics EDPE-2003, High Tatras, Slovakia, 2003.

[10] Y. Toshima, N. Ando and H. Hashimoto, "Information Display System using Active Projector in Intelligent Space -Integration of distributed devices based on RTMiddleware," in The 12th International Sysmposium on Artificial Life and Robotics, 2007.

[11] V. Devecseri, A. Dóka, J. Molnár and P. Tamás, “An ethological motion capture system," in 2011 IEEE 12th International Symposium on Computational Intelligence and Informatics (CINTI), Budapest, 2011. 